\title{
REVIEWS
}

\section{Factors Affecting Resident Satisfaction in Continuity Clinic-a Systematic Review}

\author{
J. Stepczynski, MD' , S. R. Holt, MD, MS' , M. S. Ellman, MD², D. Tobin, MD², and Benjamin R. Doolittle, \\ $M D, M D N^{3}$ \\ 'Department of Internal Medicine, Waterbury Hospital, Waterbury, CT, USA; ${ }^{2}$ Department of Internal Medicine, Yale University School of Medicine, \\ New Haven, CT, USA; ${ }^{3}$ Internal Medicine and Pediatrics, Department of Internal Medicine, Yale University School of Medicine, New Haven, CT, USA.
}

PURPOSE: In recent years, with an increasing emphasis on time spent in ambulatory training, educators have focused attention on improving the residents' experience in continuity clinic. The authors sought to review the factors associated with physician trainee satisfaction with outpatient ambulatory training.

METHODS: A systematic literature review was conducted for all English language articles published between January 1980 and December 2016 in relevant databases, including Medline (medicine), CINAHL (nursing), PSYCHinfo (psychology), and the Cochrane Central Register of Controlled Clinical Trials. Search terms included internship and residency, satisfaction, quality of life, continuity of care, ambulatory care, and medical education. We included studies that directly addressed resident satisfaction in the ambulatory setting through interventions that we considered reproducible.

RESULTS: Three hundred fifty-seven studies were reviewed; 346 studies were removed based on exclusion criteria with 11 papers included in the final review. Seven studies emphasized aspects of organizational structure such as block schedules, working in teams, and impact on resident-patient continuity (continuity between resident provider and patient as viewed from the provider's perspective). Four studies emphasized the importance of a dedicated faculty for satisfaction. The heterogeneity of the studies precluded aggregate analysis.

CONCLUSIONS: Clinic structures that limit inpatient and outpatient conflict and enhance continuity, along with a dedicated outpatient faculty, are associated with greater resident satisfaction. Implications for further research are discussed.

J Gen Intern Med 33(8):1386-93

DOI: $10.1007 / \mathrm{s} 11606-018-4469-8$

(c) Society of General Internal Medicine 2018

\section{INTRODUCTION}

Ambulatory care training during medicine residency is a foundational experience in the formation of physicians. This training provides an opportunity for residents to forge longitudinal relationships with patients, experience the natural history of

Received October 19, 2017

Revised February 22, 2018

Accepted April 18, 2018

Published online May 7, 2018 chronic disease, and develop one's personal practice style in a supervised environment. Though much of a physician's future practice in both primary and specialty care will occur in the outpatient setting, ambulatory training, as compared with inpatient training, has historically been perceived by residents as undervalued and underemphasized. ${ }^{1,2}$ Residents are often frustrated by the tension between simultaneous and conflicting inpatient and outpatient responsibilities. ${ }^{2,3}$ The complexities of the overall resident schedule (i.e., core inpatient rotations, electives, vacation and outpatient rotations) also creates difficulties for constructing resident schedules in the clinic, and thus affects resident continuity with their patients. Furthermore, patients followed within a residency continuity clinic tend to have a higher prevalence of significant comorbid medical and psychiatric illness compared with a typical general practice, ${ }^{4,5}$ Such complexity demands the most high-functioning, wellresourced practice settings. Many residency ambulatory continuity practices are poorly equipped to address the needs of these complex populations. This burden of patient complexity, coupled with often under-resourced practice settings, contributes to trainee disillusionment with primary care. ${ }^{5,6}$

In 2009, the Accreditation Council of Graduate Medical Education (ACGME) defined standards and minimum requirements for outpatient training in the core primary care specialties. $^{7}$ Specifically, the ACGME requires that residents complete a minimum of 130 half-day clinic sessions over 30 months of residency training scheduled in a fashion that minimizes the conflict between inpatient and outpatient rotations. With an increasing emphasis on time spent in ambulatory training, educators have focused attention on improving the residents' experience in continuity clinic. ${ }^{1,8,9}$ While there has been much speculation about the most prudent approach to bolster resident satisfaction in primary care, the best tactic to accomplish this objective remains unclear.

In this systematic review, we sought to identify the specific and reproducible features of the residency continuity clinic experience that influence resident satisfaction.

\section{METHODS}

Four databases were searched for relevant studies from January 1980 to December 2016 from related fields: Medline 
(medicine), CINAHL (nursing), PSYCHinfo (psychology), and the Cochrane Central Register of Controlled Clinical Trials. Search terms included internship and residency, satisfaction, quality of life, continuity of care, ambulatory care, and medical education. Standard protocol was followed for Preferred Reporting Items for Systematic Reviews and MetaAnalysis (PRISMA). ${ }^{10}$ Two researchers independently employed these search criteria. The title and abstract of each article was screened for relevancy. Relevant articles were then discussed among all authors to evaluate for bias.

We included studies focused specifically on resident satisfaction in the clinic, either through an intervention or cohort survey. Studies were included if the innovation could be replicated in other institutions, based on the consensus of the reviewers. We excluded studies that did not directly assess satisfaction with the continuity clinic experience, even if they addressed satisfaction with an educational intervention or novel curriculum. Studies that failed to include the resident physician in their clinical or operational variables were also excluded, even if clinic satisfaction was assessed.

\section{RESULTS}

The electronic search identified 534 articles. After excluding duplicates, 357 studies were evaluated. Two studies were removed after initial screening: $79(22 \%)$ review articles and $36(10 \%)$ opinion pieces failed to meet the inclusion criteria, 75 (21\%) clinical/operational studies were not focused on the ambulatory training setting, and $21(6 \%)$ studies were nonmedical in scope (Fig. 1). We excluded an additional 137 papers after a more detailed review: $114(32 \%)$ described an educational or curricular innovation that did not focus on resident satisfaction with their continuity clinic experience, and $23(6 \%)$ discussed ambulatory training clinical operations in residency programs but did not include evaluating resident satisfaction. Ultimately, 11 studies were included for this review (Fig. 1).

\section{Description of Studies}

Of the 11 studies included for analysis, 9 were from internal medicine residency programs, 1 from pediatrics and 1 from family medicine (Fig. 1). While most studies evaluated a single residency program (Barnett et al., ${ }^{11}$ Harrison et al., ${ }^{12}$ Chaudhry et al., ${ }^{13}$ Hochman et al., ${ }^{14}$ Lofgren et al., ${ }^{15}$ Roth et al., ${ }^{16}$ Warm et al., ${ }^{17}$ Wieland et al. ${ }^{18}$ ), two studies each evaluated three residency programs (Sisson et al. ${ }^{19}$ and Percoralo et al. ${ }^{1}$ ) and one study evaluated 36 internal medicine programs (Serwint et al. ${ }^{20}$ ). There was no uniform survey tool used to assess satisfaction among this group of studies. Broadly, the 11 included studies can be divided into two categories: interventional studies that measured changes in resident satisfaction in response to clinic reorganization $(n=7)$ and crosssectional studies that explored the association of specific factors with resident satisfaction $(n=4)$.

\section{Interventional Studies on Scheduling and Reorganization}

Programmatic restructuring interventions described by Harrison et al., ${ }^{12}$ Chaudhry et al., ${ }^{13}$ Warm et al.,, ${ }^{17}$ and Lofgren and Mladenovic ${ }^{15}$ all resulted in statistically significant increases in resident satisfaction. In contrast, the restructuring intervention described by Wieland et al. ${ }^{18}$ did not influence resident satisfaction. Similarly, reorganization efforts isolated to the ambulatory training setting (Roth et al. ${ }^{16}$ and Hochman et al. ${ }^{14}$ ) did not result in increased resident satisfaction.

The models proposed in the interventional studies varied significantly. In their 1990 paper, Lofgren and Mladenovic ${ }^{15}$ describe creating what we would now call the "traditional" half-day per week clinic model. In this model, PGY2 and PGY3 residents scheduled patients into one afternoon clinic session per week no matter the inpatient rotation. In contrast to this traditional model, Harrison et al. ${ }^{12}$ and Chaudhry et al. ${ }^{13}$ created a $3: 1$ and $4: 1$ scheduling model, respectively, representing the ratio of consecutive inpatient to outpatient weeks assigned to residents. During the 1-week ambulatory portion, residents spent each morning in continuity clinic and each afternoon in a subspecialty clinic. Residents were not scheduled in continuity clinics outside of the ambulatory block, thus there was a clear separation of inpatient and outpatient responsibilities. Taking this model further, Warm et al. ${ }^{17}$ created an ambulatory "long-block" (described below) and divided residents into ambulatory group practices. For the first 16 months of residency, continuity clinics occurred in the traditional clinic model before residents transitioned to the ambulatory long-block in month 17. During the long-block, which lasted for 12 months, residents were immersed into the ambulatory environment by participating in continuity clinic and subspecialty clinic, as well as educational and practice management sessions. During the long block, they had no inpatient responsibilities.

Each of these interventions led to the increased perception of resident-patient continuity (Resident-Patient Continuity from Resident provider perspective, RPC-R) (Harrison et al., ${ }^{12}$ Chaudhry et al., ${ }^{13}$ Warm et al., ${ }^{17}$ and Lofgren and Mladenovic ${ }^{15}$ ). Apart from Lofgren and Mladenovic, they all demonstrated decreased tension between inpatient and outpatient responsibilities. Harrison et al. ${ }^{12}$ also demonstrated an improvement in resident satisfaction with exposure to subspecialty medicine.

In redesigning their schedule to a $4: 4$ structure (4 weeks inpatient: 4 weeks outpatient), Wieland et al. ${ }^{18}$ separated inpatient and outpatient responsibilities and focused on increasing preceptor-resident continuity, creating resident teams, and intensifying the ambulatory curriculum. Because of this reorganization, patient access and resident-patient panel size both increased. In contrast to the other studies, however, Wieland et al. ${ }^{18}$ documented a decrease in RPC-R. Resident satisfaction with their ambulatory training experience did not change. 


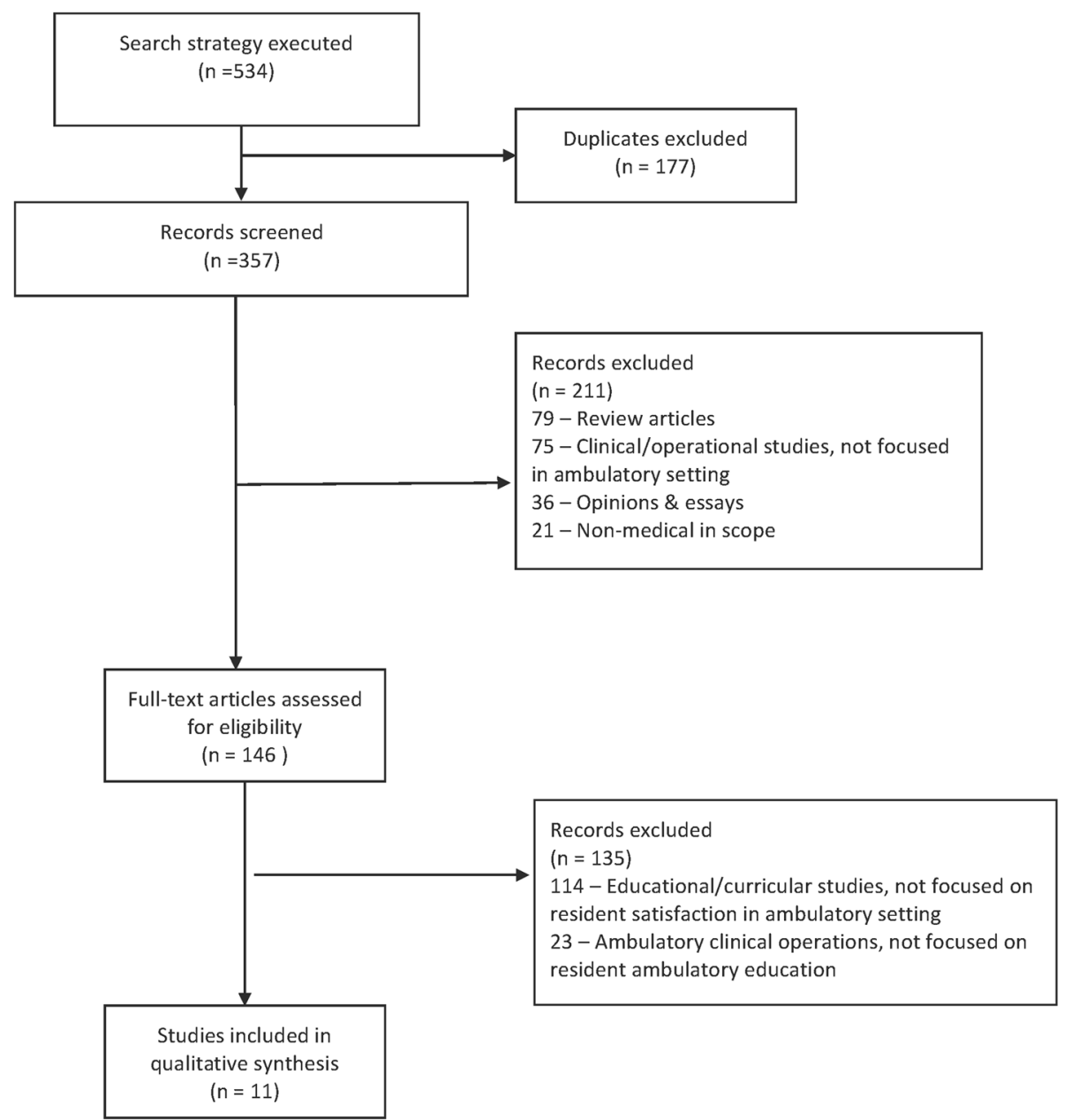

Fig. 1 PRISMA search strategy

Roth et al. ${ }^{16}$ examined the impact of organizing a family medicine residency clinic into three distinct teams, each team composed of two faculty members, eight residents, and three medical assistants. Improving preceptor-resident continuity by manipulating the faculty schedules was a point of emphasis. The program initiated team meetings during which they reviewed team performance and discussed increasing team member engagement. Using the Organizational Environment Assessment and Learning Environment Assessment surveys, Roth et al. demonstrated improved satisfaction among faculty and staff but not among residents. Hochman et al. ${ }^{14}$ reorganized their internal medicine residency clinic into a patient-centered medical home (PCMH). By doing so, they introduced a resident triage role in the call center, increased the number of urgent appointment slots per day, and increased case management and care coordination resources. These changes lead to increased patient satisfaction but did not lead to a statistically significant increase in resident satisfaction.

\section{Cross-sectional Studies}

Of the cross-sectional studies reviewed, three surveyed multiple residency programs (Peccoralo et al., ${ }^{1}$ Serwint et al., ${ }^{20}$ Sisson et al. ${ }^{19}$ ) and analyzed factors that correlated with resident satisfaction (Serwint et al. ${ }^{20}$ ), resident valuation of their continuity experience (Sisson et al. ${ }^{19}$ ), or the likelihood of pursuing a career in general internal medicine (Peccoralo et al. ${ }^{1}$ ). The fourth (Barnett et al. ${ }^{11}$ ) explored factors that correlated with residents' satisfaction with a patient interaction in their continuity clinics using a post-encounter questionnaire.

Two cross-sectional studies (Serwint et al. ${ }^{20}$ and Sisson et al. ${ }^{19}$ ) noted a correlation between a resident's identification of her preceptor as a role model with an increased likelihood of satisfaction with the ambulatory training experience. A preceptor's perceived communication of clinical reasoning, receptiveness to questions about management, enthusiasm, and effectiveness in generating a differential diagnosis and managing medical issues were all behaviors that correlated with being a good role model (Sisson et al. ${ }^{19}$ ). In both studies, a resident's rating of their preceptor as a good role model correlated most strongly with satisfaction.

Continuity of care with patients also emerged as an important factor for satisfaction. Using a post-encounter questionnaire that assessed overall satisfaction with the encounter, continuity with patient, and type of problems addressed during the visit, Barnett et al. ${ }^{11}$ demonstrated that RPC-R increased 
satisfaction with complex patients previously associated with poor post-visit resident satisfaction (e.g., patients with pain disorders or complicated psychiatric disease). Peccoralo et al. ${ }^{1}$ demonstrated a correlation between feeling very satisfied with the continuity of the resident provider-patient relationship and likelihood of choosing a career in general internal medicine.

\section{DISCUSSION}

The purpose of this systematic review was to identify specific, modifiable factors that a residency program could use as a roadmap to improve the ambulatory training experience of trainees. The main themes that emerged as factors that may influence resident satisfaction were organizational factors that promoted both the separation of inpatient and outpatient responsibilities and RPC-R, and faculty characteristics, such as preceptors viewed as role models (Table 1).

First, scheduling continuity clinics in discrete blocks separate from inpatient rotations rather than during inpatient rotations, with the intent to eliminate tension between inpatient and ambulatory responsibilities, resulted in increased resident satisfaction. The superiority of the block schedule over a traditional or mixed scheduling scheme - in regard to limiting the conflict between inpatient and outpatient responsibilities - was also noted in another paper by Frances et al., ${ }^{22}$ a cross-sectional study of 12 internal medicine residency programs. Second, interventions that increased RPC-R resulted in increased resident satisfaction. In contrast, when residents' perception of continuity with their patients decreased, overall resident satisfaction did not improve even if the tension between inpatient and outpatient responsibilities was simultaneously eliminated. We hypothesize that RPC-R decreases when the reorganizational intervention results in an increase in resident panel size, crossing a threshold that compromises continuity. Third, residents identified having dedicated, high-performing faculty committed to ambulatory education as a critical influence. Physician role models, which are enthusiastic, receptive, and effective in managing medical issues, created a quality education milieu (Table 2).

Our analysis suggests that interventions designed to decrease the tension between simultaneous inpatient and outpatient time demands, as well as changes that increase RPC-R, are likely to result in an improved ambulatory learning environment, particularly when they occur together. Such interventions must carefully consider their effect on panel size, clinic frequency, and the total number of clinics as all three of these factors affect trainee perception of continuity. ${ }^{21}$

\section{LIMITATIONS}

There are several limitations to this study. First, our literature search failed to identify relevant studies from many predominantly outpatient medical specialties (e.g., dermatology, pulmonology, hematology/oncology, and obstetrics/gynecology). Similarly, we did not identify appropriate articles from other healthcare fields such as nursing or psychology, despite our open search criteria. It is noteworthy that the studies identified by our review were all from programs in "primary care" fields (pediatrics, internal medicine, family medicine); we suspect this reflects the importance such programs place on creating a positive ambulatory experience and the desire to influence the future career choice of trainees. This narrower than expected cohort suggests the need for further investigation. Second, we found the identified studies to have heterogeneous methodology, with no standardized approach to measuring trainee satisfaction with their ambulatory training experience. As a result, it is difficult to assess the quantitative impact of the described changes on the resident experience. We were able to use $p$ values to assess significance of the findings, but the "amount" of change in resident satisfaction is difficult to quantify. Finally, most studies evaluated only one ambulatory training site, so our findings may not be generalizable to programs with more than one ambulatory continuity clinic.

\section{IMPLICATIONS FOR RESIDENCY EDUCATION}

Our systematic review identified a number of complex and actionable interventions for residency programs interested in improving resident satisfaction in ambulatory training. First, programs should structure their ambulatory training in a way that minimizes competition between inpatient and outpatient responsibilities and enhances continuity between resident providers and their patients. Second, programs must invest in recruiting, developing, and retaining role model faculty devoted to primary care. We believe such faculty should be knowledgeable about outpatient resources, should mentor trainees, and must be given enough recognition and influence within training programs for residents to perceive ambulatory training as a programmatic priority. Residents who identify such faculty as role models are most likely to be satisfied with their ambulatory training experience. We hypothesize that by exposing residents to a positive and engaging educational experience and improving satisfaction with their ambulatory training, more trainees will elect to pursue a career in primary care. As educators, our hope is that by ensuring the above modifications are widely adopted, and resident satisfaction in continuity clinic is improved, the enhanced overall resident clinic experience will influence more residents to pursue a career in primary care.

Robust and meaningful ambulatory training is a key ingredient for the development of the next generation of providers. At a time when there is a critical need for primary care physicians, there is urgency to get ambulatory education right. By investing in our faculty, minimizing inpatient/outpatient conflict, and developing resident: patient continuity, we move 


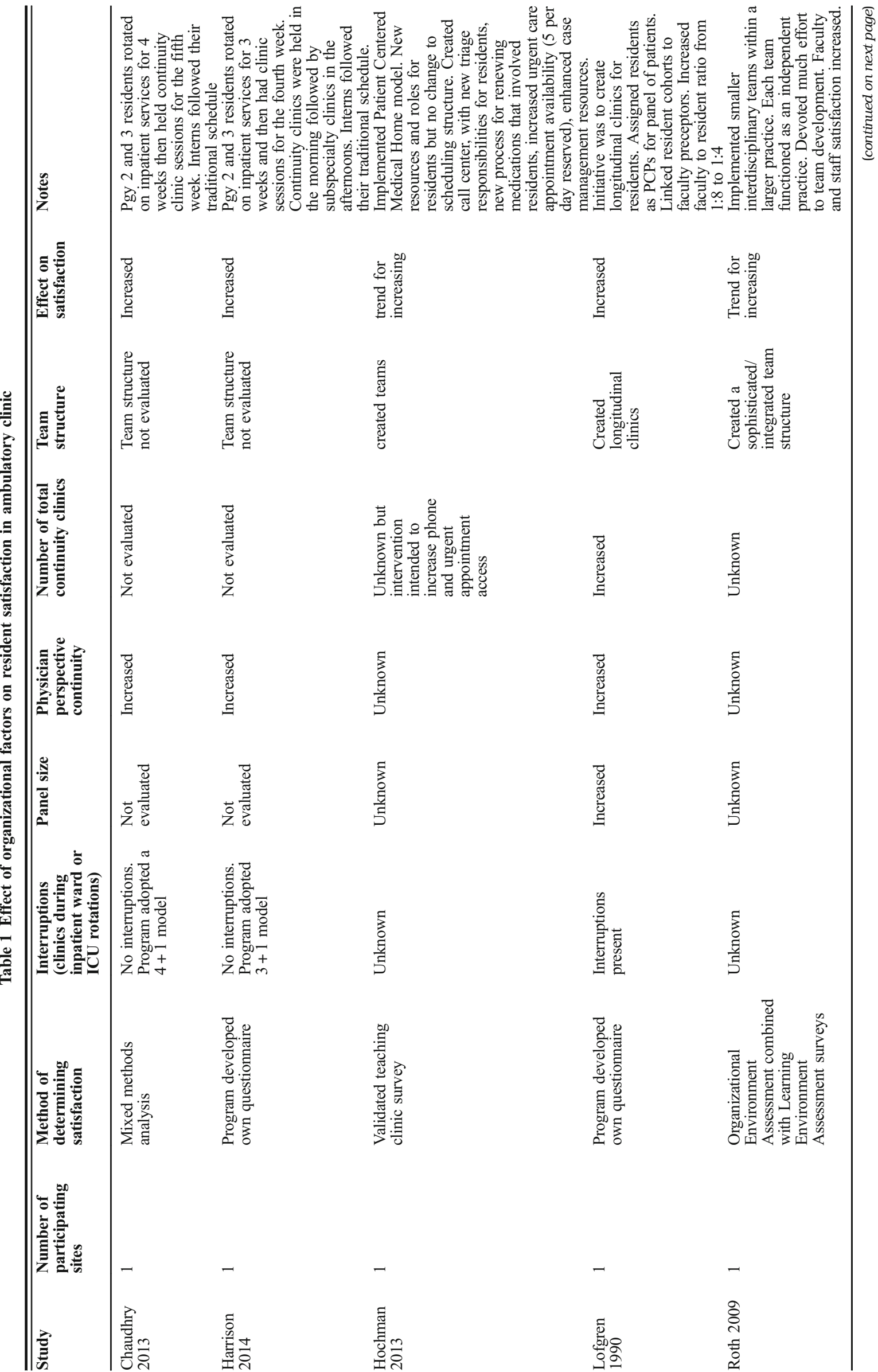




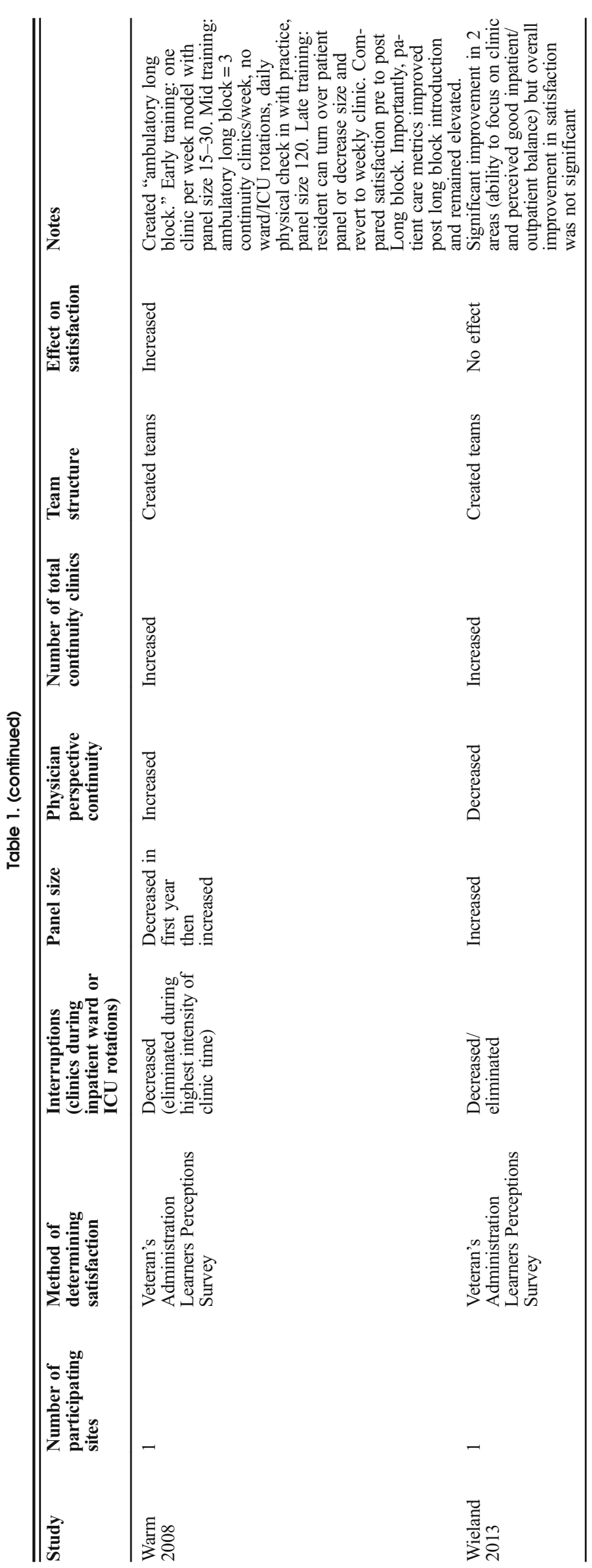




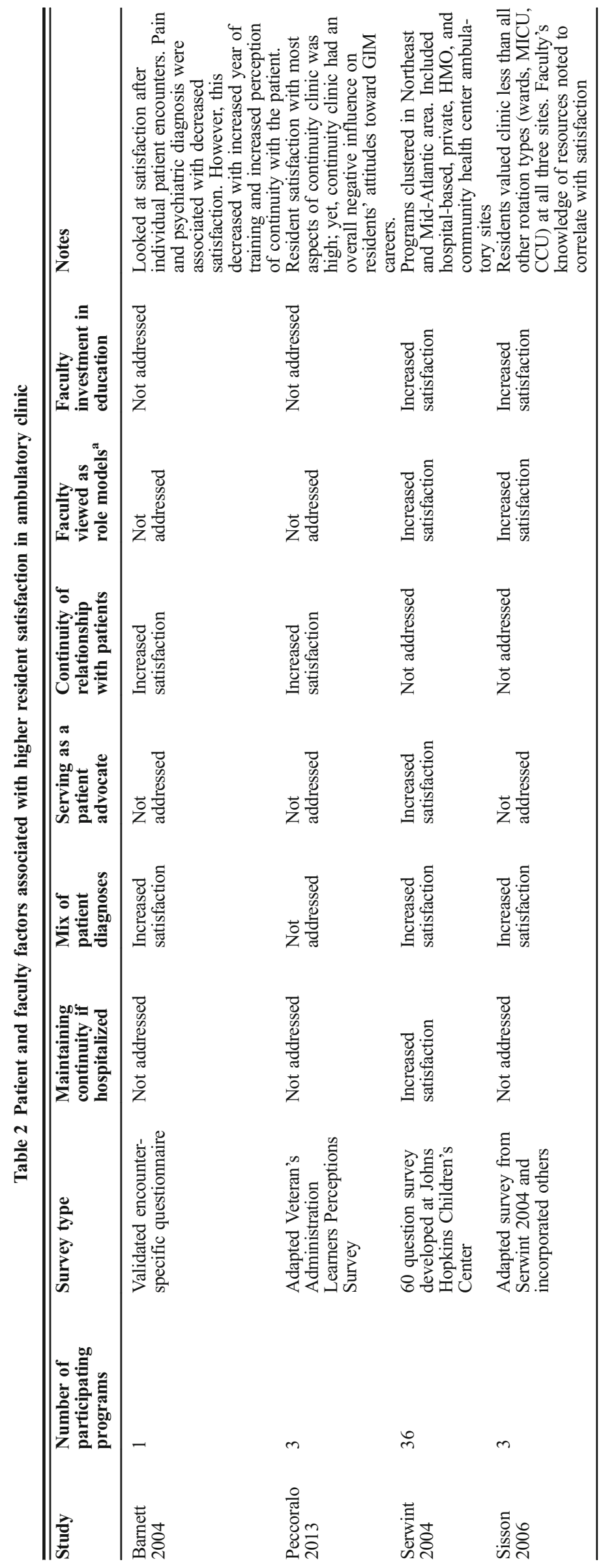


one step closer to meeting the needs of our patients, communities, and nation.

Acknowledgments: The authors extend thanks and appreciation for Dr. Patrick O'Connor for his support and encouragement.

Corresponding Author: Benjamin R. Doolittle, MD, MDIV; Internal Medicine and Pediatrics, Department of Internal Medicine Yale University School of Medicine, 1074 LMP, PO Box 8030, New Haven, CT 06520-8030, USA (e-mail: Benjamin.doolittle@yale.edu).

\section{Compliance with ethical standards:}

Conflict of interest: The authors declare that they have no conflict of interest to declare.

\section{REFERENCES}

1. Peccoralo LA. Tackett S, Ward L, et al. Resident satisfaction with continuity clinic and career choice in general internal medicine. J Gen Intern Med 2013;28(8):1020-1027.

2. Meyers FJ, Weinberger SE, Fitzgibbons JP, Glassroth J, Duffy FD, Clayton CP. Redesigning residency training in internal medicine: the consensus report of the Alliance for Academic Internal Medicine Education Redesign Task Force. Acad Med 2007;82(12):1211-1219.

3. Salerno SM, Faestel PM, Mulligan T, Rosenblum MJ. Disruptions and satisfaction in internal medicine resident continuity clinic differ between inpatient and outpatient rotations. Teach Learn Med 2007;19(1):30-34.

4. Didden DG, Philbrick JT, Schorling JB. Anxiety and depression in an internal medicine resident continuity clinic: difficult diagnoses. Int $\mathrm{J}$ Psychiatry Med 2001;31(2):155-167.

5. Keirns CC, Bosk CL. Perspective: the unintended consequences of training residents in dysfunctional outpatient settings. Acad Med 2008;83(5):498-502.

6. Weinberger SE, Smith LG, Collier VU. Redesigning training for internal medicine. Ann Intern Med 2006;144(12):927-932.

7. http://www.acgme.org/Portals/0/PFAssets/ProgramRequirements/ 140_internal_medicine_2017-07-01.pdf?ver=2017-06-30-083345-723. Accessed 09/26/2017

8. Wieland ML, Halvorsen AJ, Chaudhry R, Reed DA, McDonald FS, Thomas KG. An evaluation of internal medicine residency continuity clinic redesign to a 50/50 outpatient-inpatient model. J Gen Intern Med 2013;28(8): 1014-1019.
9. Cronholm PF, Klusartiz H, Nguyen GT, Kellom K, Kearney M, MillerDay M, Gabbay R. Resident engagement in the patient-centered medical homs. Fam Med 2016;48(8):603-612.

10. Moher D, Liberati A, Tetzlaff J, Altman DG, The PRISMA Group. Preferred Reporting Items for Systematic Reviews and Meta-Analyses: The PRISMA Statement. PLoS Med 2009;6(6): e1000097.

11. Barnett DR, Bass PF, Griffith CH, Caudill TS, Wilson JF. Determinants of resident satisfaction with patients in their continuity clinic. J Gen Intern Med 2004;5:456-459.

12. Harrison JW, Ramaiya A, Cronkright P. Restoring emphasis on ambulatory internal medicine training - the 3:1 model. J Grad Med Educ. 2014;694):724-725.

13. Chaudhry SI, Balwan S, Firedman KA, et al. Moving forward in GME reform: a $4+1$ model of resident ambulatory training. J Gen Intern Med 2013;28(8):1100-1104.

14. Hochman ME, Asch S, Jibilian A, et al. Patient-centered medical home intervention at an internal medicine resident safety-net clinic. JAMA Intern Med 2013;173(18):1694-1701.

15. Lofgren RP, Mladenovic J. How reorganizing a general medicine clinic affected residents' and patients' satisfaction. Acad Med 1990;65(9):604608

16. Roth LM, Markova T, Monsur JC, Severson RK. Effects of implementation of a team model on physician and staff perceptions of a clinic's organizational and learning environments. Fam Med 2009;41(6):434439 .

17. Warm EJ, Schauer DP, Diers T, et al. 2008. The ambulatory long-block: an accreditation council for graduate medical education (ACGME) educational innovations project (EIP). J Gen Intern Med.23(7):921-926.

18. Wieland ML, Halvorsen AJ, Chaudhry R, Reed DA, McDonald FS, Thomas KG. An evaluation of internal medicine residency continuity clinic redesign to a 50/50 outpatient-inpatient model. J Gen Intern Med 2013;28(8):1014-1019.

19. Sisson SD, Boonyasa R, Baker-Genaw K, Silverstein J. Continuity clinic satisfaction and valuation in residency training. $\mathrm{J}$ Gen Intern Med 2007;22(12):1704-1710

20. Serwint JR, Feigelman S, Dumont-Driscoll M, Collins R, Zhan M, Kittredge D. Factors associated with resident satisfaction with their continuity experience. Ambul Pediatr 2004;4(1):4-10.

21. Francis MD, Zahnd WE, Varney A, Scaife SL, Francis ML. Effect of number of clinics and panel size on patient continuity for medical residents. J Grad Med Educ 2009;1(2):310-315.

22. Francis MD, Thomas $\mathbf{K}$, Langan M, Smith A, Drake S, Gwisdalla KL, Jones RR, Juuan KA, Nabors C, Pereira A, Rosenblum M, Varney A Warm E, Ortiz $\mathbf{M}$, Clinic design, key practice metrics, and resident satisfaction in Internal Medicine continuity clinics: findings of the educational innovations project ambulatory collaborative. J Grad Med Educ. 2014;6 (2):249-255 